\title{
Avaliação de Softwares Educacionais: Uma Abordagem Mais Inclusiva para Alunos com Transtorno do Espectro Autista
}

\author{
Uilliam P. Oliveira, Wesley B. Thereza \\ FALECT - Universidade do Estado de Mato Grosso (UNEMAT) \\ Rua Santa Rita, 128 - 78.780-000 - Alto Araguaia - MT - Brasil \\ \{uilliam.oliveira, wesley\}@unemat.br
}

\begin{abstract}
Students with Autism have difficulties in developing cognitive skills and acquiring new knowledge due to their intellectual disabilities. This paper aimed to understand Autistic Spectrum Disorder (ASD), its intervention techniques and to verify whether the educational software evaluation approaches in the literature allow us to evaluate a product for students with Special Educational Needs (SEN) and can be used by professionals of education in the teaching-learning process. The result was an adapted, more inclusive and easy-to-apply checklist, enhancing the learning and development of children within the spectrum.

Resumo. Os alunos com Autismo têm dificuldades em desenvolver habilidades cognitivas e adquirir novos conhecimentos devido a suas deficiências intelectuais. Este artigo teve como objetivo compreender o Transtorno do Espectro Autista (TEA), suas técnicas de intervenção e verificar se as abordagens de avaliação de software educacional na literatura nos permitem avaliar um produto para estudantes com Necessidades Educacionais Especiais (NEE) e podem ser usadas por profissionais da educação no processo de ensino-aprendizagem. O resultado foi uma checklist adaptada, mais inclusiva e fácil de aplicar, aprimorando o aprendizado e o desenvolvimento das crianças dentro do espectro.
\end{abstract}

\section{Introdução}

A inclusão escolar, social e digital de crianças com Necessidades Educativas Especiais (NEE) nas escolas está dependendo cada vez mais de recursos da computação, que possuem potencial para serem utilizadas como recurso pedagógico, favorecendo o nível de qualidade do ensino-aprendizagem (Valente, 1998). O problema está tanto na adoção das ferramentas pelos profissionais da educação, visto que há - por parte deles - uma insegurança ocasionada pela falta de treinamento para com as novas tecnologias (Buckingham, 2010), quanto na avaliação de tais softwares para serem aplicados em sala de aula (Silva et al., 2016).

O presente estudo pesquisou, analisou e discutiu o atual cenário das ferramentas de avaliação de Softwares Educacionais (SEs), se estas permitem a adoção de produtos informatizados para alunos com o Transtorno do Espectro Autista (TEA), e se são de fácil aplicação e entendimento para profissionais da educação que venham utilizá-las como instrumentos pedagógicos. Como resultado, adaptamos uma das analisadas, tornando-a mais inclusiva e de fácil aplicação. 
VIII Congresso Brasileiro de Informática na Educação (CBIE 2019)

Anais do XXV Workshop de Informática na Escola (WIE 2019)

Este resumo está dividido em dez seções, incluindo essa. Logo na parte 2 é apresentado a metodologia utilizada. Posteriormente na 3 temos a exploração das abordagens. Na 4 temos as considerações do estudo e trabalhos futuros, e por fim temos as referências.

\section{Metodologia}

Essa pesquisa seguiu uma abordagem exploratória com elementos de um mapeamento sistemático, por realizar o levantamento bibliográfico das técnicas e proporcionar uma visão geral sobre as abordagens e sua relação com SEs e seus públicos-alvos. Identificamos as abordagens, analisamo-las dentro do contexto da educação especial, adaptamos e validamos empiricamente uma nova abordagem de acordo com pesquisa bibliográfica sobre o TEA, a análise dos paradigmas de intervenção e o referencial teórico.

Primeiramente foi feito um mapeamento sistemático nos anais de eventos que possuem trilha ou são destinados ao tema de informática na educação: TISE (Conferência Internacional sobre Informática na Educação) e SBIE (Simpósio Brasileiro sobre Informática na Educação), utilizando palavras-chaves (strings de busca): ("avaliação" OR "assessment" OR "evaluación" OR "evaluatión" OR "educação especial” OR "special education", "educación especial" OR "software educacional" OR "educational software" OR “checklist" OR "lista de verificación” ) AND ("TEA" OR “ASD” OR "autismo" OR "autism", OR "autista" OR "autistic"), que compreendeu publicações de 2016 a 2018, admitindo artigos resumidos (short papers) e completos (full papers) escritos em Português, Inglês e Espanhol.

Devido ao excesso de trabalhos com análises bibliográficas dos mesmos métodos foi necessário fazer um refinamento, de acordo com esses critérios de exclusão: publicações sobre revisão sistemática sobre o Autismo; estudos direcionados a desenvolvedores; estudos duplicados; estudos que apresentam soluções de intervenção sem uma avaliação e Estudos sobra o TEA que fogem da temática da educação - Tabela 1. A partir desse ponto, iniciamos a abordagem exploratória dos trabalhos selecionados e adaptamos uma tabela comparativa dos mesmos, a fim de verificar se as abordagens de avaliação abrangem qualidades pedagógicas, de interface e de software em um SE para educandos dentro de espectro.

\begin{tabular}{|c|c|c|}
\hline Eventos & Trabalhos encontrados & Trabalhos selecionados \\
\hline TISE & 31 & 03 \\
\hline SBIE & 63 & 05 \\
\hline
\end{tabular}

Tabela 1: Relação de trabalhos encontrados. Fonte: Autores.

\section{Exploração das Abordagens}

Nesta seção apresentamos a exploração das abordagens, relacionando-as de acordo com as técnicas de intervenção e referencial teórico apresentados no estudo de Oliveira e Thereza (2019), para que possa ser verificado qual paradigma se encaixaria ou seria melhor adaptado para avaliar tais SEs. Primeiramente, salientamos pontos primordiais que devem ser levados em consideração em um SE para educandos com TEA, que são:

- Base pedagógica: É relevante a questão pedagógica dos SEs serem elaborados seguindo os métodos ou as teorias pela qual o sujeito aprende, como ele se apropria e constrói o conhecimento (Vieira, 1999, Silva et al., 2016); 
VIII Congresso Brasileiro de Informática na Educação (CBIE 2019)

Anais do XXV Workshop de Informática na Escola (WIE 2019)

- Utilização de mecanismos/abordagens utilizadas nas esferas clínicas e/ou educacionais: Refere-se à atenção dos métodos de avaliação de SE - independente se estes forem direcionados a um público alvo ou não - estão verificando meios ou técnicas para potencializar o processo de ensino-aprendizagem;

- Adaptabilidade: Um SE adaptável ao usuário permite o mesmo trabalhar em seu próprio ritmo e nível de habilidade, maximizando seus pontos fortes e minimizando os pontos de dificuldades, de forma que ajude na progressão da aprendizagem (Zakari et al., 2014).

- Acessibilidade: A interface permite uma melhor comunicabilidade dos produtores do sistema aos usuários, desta forma, permitindo que o software seja utilizado e entendido por qualquer pessoa, seja pelo o usuário em busca do conhecimento ou um mediador que venha adotá-la como ferramenta de ensino;

- Portabilidade: Educandos com TEA possuem limitada habilidade de generalização e carecem de estímulos constantes (Gomes e Souza, 2016, Kwee, 2006), portanto um SE que permita que a criança continue aprendendo o conteúdo - seja conteúdo acadêmico ou atividades da vida diária - de maneira variada e lúdica fora do contexto escolar e/ou clínica pode favorecer uma melhor seu desenvolvimento. A viabilidade de um SE multiplataforma pode conduzir o educando a melhores formas entender o mundo que o cerca;

- Avaliação: As Tecnologias Digitais da Informação e Comunicação trazem um novo paradigma educacional e, com novas formas de ensinar, vem também novas formas de avaliar. Essas novas ferramentas devem permitir o registro e extração de dados das atividades executadas nele, a fim de análises posteriormente pelos educadores e outros profissionais que acompanham o desenvolvimento do educando.

Após a definição dos requisitos a serem verificados nas abordagens, iniciamos o processo de exploração das mesmas. A Figura 1 traz uma visão panorâmica sobre as técnicas de avaliação e os requisitos essenciais anteriormente definidos. Para simplificação das técnicas encontradas no estudo de Oliveira e Thereza (2019), foram atribuídos um identificador para cada uma delas, em que "A" significa "Abordagem" e "N" o número de identificação da mesma.

\begin{tabular}{|c|c|c|c|c|c|c|}
\hline Id & Base pedagógica & $\begin{array}{l}\text { Utilização de } \\
\text { mecanismos }\end{array}$ & Adaptabilidade & Acessibilidade & Portabilidade & Avaliação \\
\hline A01 & $\checkmark$ & & & $\checkmark$ & & \\
\hline A02 & $\checkmark$ & & $\checkmark$ & $\checkmark$ & $\checkmark$ & \\
\hline A03 & $\checkmark$ & & $\checkmark$ & $\checkmark$ & & $\checkmark$ \\
\hline A04 & $\checkmark$ & & $\checkmark$ & $\checkmark$ & & \\
\hline A05 & $\checkmark$ & & $\checkmark$ & $\checkmark$ & & \\
\hline A06 & $\checkmark$ & & & $\checkmark$ & & $\checkmark$ \\
\hline \multicolumn{7}{|l|}{ A07 } \\
\hline A08 & $\checkmark$ & $\checkmark$ & & & & \\
\hline A09 & & $\checkmark$ & & $\checkmark$ & & \\
\hline A10 & $\checkmark$ & & & $\checkmark$ & & \\
\hline A11 & $\checkmark$ & $\checkmark$ & $\checkmark$ & $\checkmark$ & $\checkmark$ & $\checkmark$ \\
\hline A12 & $\checkmark$ & & & & & \\
\hline A13 & $\checkmark$ & & $\checkmark$ & $\checkmark$ & $\checkmark$ & \\
\hline
\end{tabular}

Figura 1: Panorama das abordagens em relação aos requisitos. Fonte: Autores. 
VIII Congresso Brasileiro de Informática na Educação (CBIE 2019)

Anais do XXV Workshop de Informática na Escola (WIE 2019)

Foi notório que a abordagem A11 mesmo não sendo direcionada para educandos com necessidades educativas especiais, apresentou-se bem tanto no trabalho de Oliveira e Thereza (2018) quanto no presente estudo, visto que:

I. É fácil de ser entendida e aplicada por professores e por pessoas mais leigas, não necessitando de conhecimento em computação;

II. Abrange tanto aspectos pedagógicos, quanto de qualidade de interface e de software - de forma implícita;

III. Possui métricas especificas para cada categoria de software, valorizando as propriedades distintas de cada um.

Portanto, para deixá-la mais inclusiva, criamos e adaptamos algumas heurísticas com aspectos dos métodos de intervenção e da teoria sócio histórica apresentados e disponível no estudo de Oliveira e Thereza (2019), validando-a empiricamente com softwares para educandos atípicos e com SEs tradicionais, fomentando a questão: Um SE tradicional pode ser utilizado para ensinar alunos com TEA? Outras abordagens como A07 não marcou quaisquer pontos na Figura 1 por tratar-se de uma ferramenta web direcionada a especialistas e avaliadores de características de softwares. No tópico da acessibilidade, foi notório que A08 e A12 não frisam tal requisito por serem técnicas específicas focadas nas experiências dos usuários em jogos digitais educacionais.

\section{Considerações Finais e Trabalhos Futuros}

É incontestável a pluralidade das abordagens de avaliação de softwares educacionais. Todas possuem pontos semelhantes e distintos, algumas são simples de aplicar e outras necessitam de conhecimentos específicos, no entanto, cabe ao usuário verificar qual se adequa melhor a sua situação. Diante disso realizamos a presente pesquisa a fim de verificar se havia abordagens que possibilitasse a análise de softwares educacionais para educandos com Autismo e qual seria o modelo de avaliação mais indicado para classificar os softwares a serem utilizados junto a alunos com o Transtorno do Espectro Autista, e a conclusão foi a adaptação de uma checklist mais inclusiva e fácil de ser aplicada por profissionais da educação.

Para trabalhos futuros, com o intuito de elaborarmos uma intervenção baseada em tecnologia multiplataforma e gratuita, iremos: (i) Analisar os SEs pagos, para verificar e discutir sua qualidade correlacionada com os softwares gratuitos; (ii) Melhorar a checklist adaptada seguindo o guia Goal Driven Software Measurement (baseado no estudo de Almeida et al, 2018); (iii) Validá-la empiricamente e quantitativamente com a ajuda das famílias, profissionais que trabalham com esse público e dos mais recentes softwares educacionais disponíveis para download; e por último e não menos importante, (iv) Fazer um site para divulgação da checklist com detalhes sobre cada heurística nela presente e versão imprimível, para que não fique limitada apenas a pesquisadores, visto que a pesquisa em si preocupou-se com a facilitação da adoção de tais ferramentas digitais pelos educadores.

\section{Referências}

Almeida, A. et al. (2018) Indicadores para Avaliação de Software Educacional com base no guia GDSM (Goal Driven Software Measurement). Simpósio Brasileiro de Informática na Educação (SBIE), [S. 1.]. Disponível em: http://www.brie.org/pub/index.php/sbie/article/view/7950. Acesso em: 10 mar. 2019.

Buckingham, D. (2010) Cultura Digital, Educação Midiática e o Lugar da Escolarização. Educação \& Realidade, Porto Alegre. Disponível em: 
VIII Congresso Brasileiro de Informática na Educação (CBIE 2019)

Anais do XXV Workshop de Informática na Escola (WIE 2019)

https://seer.ufrgs.br/educacaoerealidade/article/view/13077/10270. Acesso em: 3 mar. 2019.

Gomes, C. G. S. e Souza, D. das G. de. (2016) Ensino de Sílabas simples, Leitura Combinatória e Leitura com Compreensão para aprendizes com Autismo Revista Brasileira de Educação Especial, [S. 1.]. Disponível em: http://www.scielo.br/scielo.php?pid=S141365382016000200233\&script=sci_abstract\&tlng=pt. Acesso em: 24 fev. 2019.

Jucá, S. C. S. (2006) A relevância dos softwares educativos na educação profissional. Ciências \& Cognição, V8: p 22-28 [online]. Revista interdisciplinar de estudos de cognição. Disponível em: http://www.cienciasecognicao.org/revista/index.php/cec/article/view/571/359. Acesso em: 02 jan. 2019.

Kwee, C S. (2006) Abordagem Transdisciplinar no Autismo: O Programa TEACCH. 110 p. Dissertação (Mestrado Profissionalizante em Fonoaudiologia) - Universidade Veiga de Almeida, Rio de Janeiro.

Oliveira, U. P. de. e Thereza, W. B. (2018) O Uso de TICs para Inclusão Social de Crianças com Transtorno do Espectro Autista. Conferência Internacional sobre Informática na Educação (TISE), Brasília, Brasil. Disponível em: http://www.tise.cl/Volumen14/TISE2018/569.pdf. Acesso em: 3 abr. 2019.

Oliveira, U. P. de; Thereza, W. B. (2019) Avaliação de Softwares Educacionais: Uma Abordagem Mais Inclusiva para Alunos com Transtorno do Espectro Autista. LatinAmerican Community of Learning Objetcs (LACLO), São Jose Del Cabo - México. No prelo.

Silva, R. S. da et al. (2016) Avaliação de Software Educativo: a complexidade de escolher uma abordagem adequada. In: Congresso Regional sobre Tecnologias na Educação (CTrl+e) Natal - RN. Disponível em: http://ceur-ws.org/Vol1667/CtrlE_2016_AC_paper_26.pdf. Acesso em: 24 fev. 2019.

Valente, J. A. (Org.). (1998) Computadores e Conhecimento: Repensando a educação. 2. ed. Campinas/SP: Unicamp/NIED. 513 p. v. 1.

Vieira, F. M. S. (1999) Avaliação de software educativo: reflexões para uma análise criteriosa. Disponível em: <https://pt.scribd.com/document/222436005/Avaliacao-deSoftware-Educativo-Reflexoes-Para-Uma-Analise-Criteriosa $>$. Acesso em: 01 mar. 2019.

Zakari, H. M. et al. (2014). A Review of Serious Games for Children with Autism Spectrum Disorders (ASD). International Conference on Serious Games Development and Applications. Springer, Cham, Berlin, Germany. Disponível em: https://www.researchgate.net/publication/291345119_A_Review_of_Serious_Games _for_Children_with_Autism_Spectrum_Disorders_ASD. Acesso em: $01 \mathrm{fev} .2019$. 